\title{
Il "Focus on" a Milano ha coronato tre anni di Road Show
}

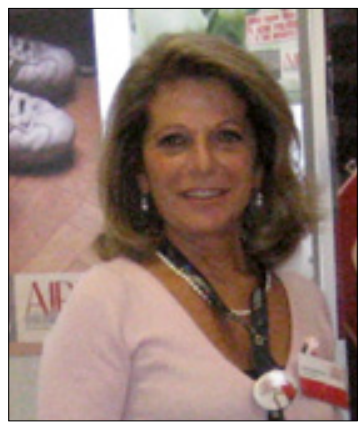

Luisa Sternfeld Pavia

Presidente

Associazione Italiana Rene

Policistico AIRP onlus

luisa.sternfeld.airp@renepolicistico.it

Cari lettori, cari amici,

dopo quindici tappe del nostro Road Show e due Tavole Rotonde, appuntamenti che hanno toccato praticamente tutta l'Italia dal 2010 a oggi, sentivamo il bisogno di fare un punto, di coronare questo ciclo di incontri con un evento più grande $\mathrm{e}$ più importante (Fig. 1).

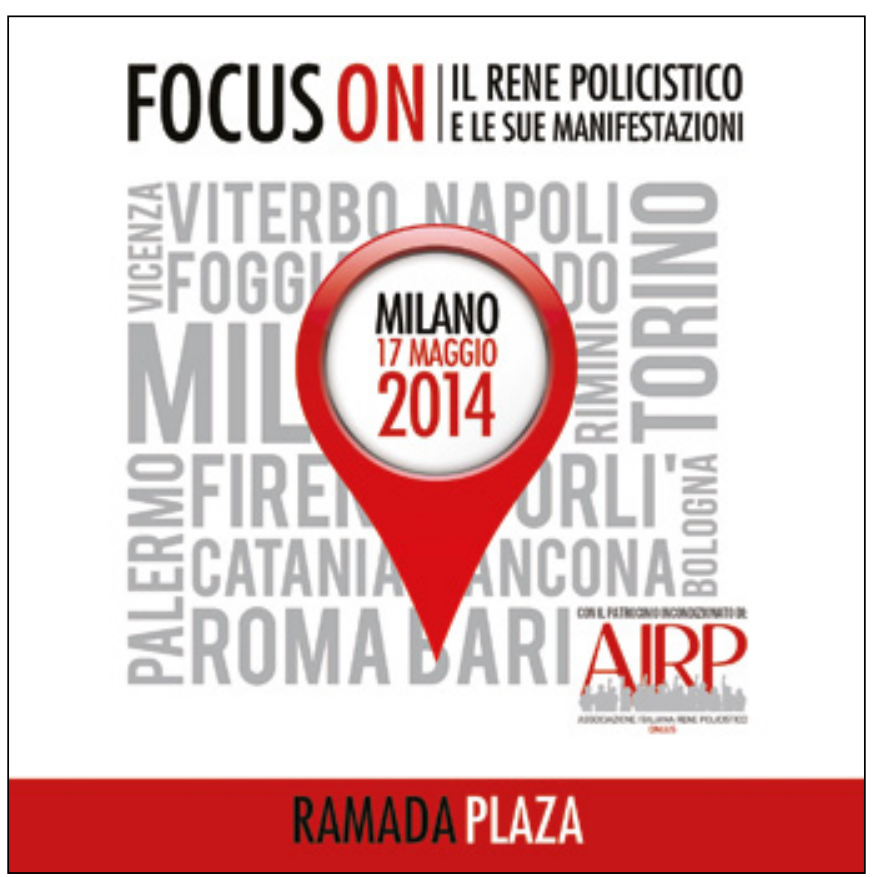

Fig. 1

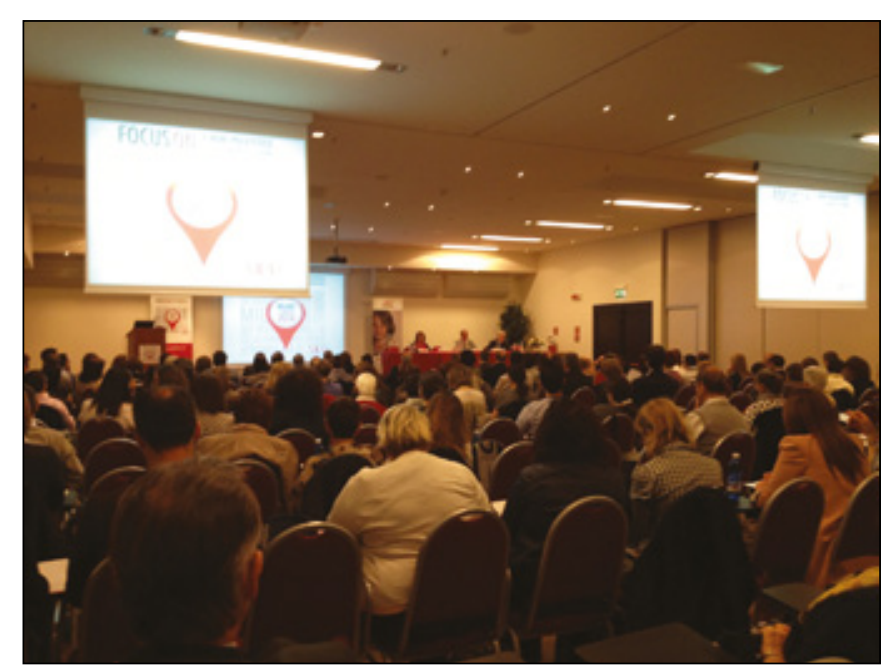

Fig. 2

Questo evento è stato "FOCUS ON. Il rene policistico e le sue manifestazioni”, Simposio Nazionale che si è svolto a Milano il 17 Maggio scorso, proprio come l'avevamo desiderato e progettato: un programma intenso con argomenti nuovi, contributi scientifici di alto livello presentati dai migliori specialisti e una grande e sentita partecipazione di pubblico: circa 180 persone, arrivate da tutta Italia (Fig. 2).

Desidero condividere con tutti coloro che non hanno potuto essere presenti una sintesi del meeting, che è stato ricco di temi e di approfondimenti di grande interesse per tutti noi.

Dopo la proiezione del video "FOCUS ON a Milano, 17 Maggio 2014: AIRP si presenta cosi" che vede ripercorrere tutte le tappe del Road Show (15 città, $16.620 \mathrm{Km}$ percorsi, 158 relatori), il FOCUS ON si è aperto con il saluto delle Autorità (per l'Ordine dei Medici di Milano la Dr.ssa Mariagrazia Manfredi, il Presidente della SIN Prof. Giovambattista Capasso, per la SIMG il Dr. Salvatore Campo, il Direttore della Facoltà di Medicina e Chirurgia dell'Università degli Studi di Milano Prof. Antonio Carrassi e per la Fondazione Telethon la Dr.ssa Alessandra Zatti).

Nella lezione magistrale per la presentazione del progetto di ricerca AIRP "Identificazione di disfunzioni metaboliche nel rene policistico: nuove opportunità terapeutiche", la Dr.ssa Alessandra Boletta ha presentato i più recenti risultati sull'utilizzo del 2-deossiglucosio (2-DG, un analogo del glucosio) sul modello sperimentale animale (topo) di rene policistico autosomico dominante.

In breve, le cellule mutanti del tubulo renale, più "affamate" di glucosio delle cellule normali, introducono il 2-DG, ma, 


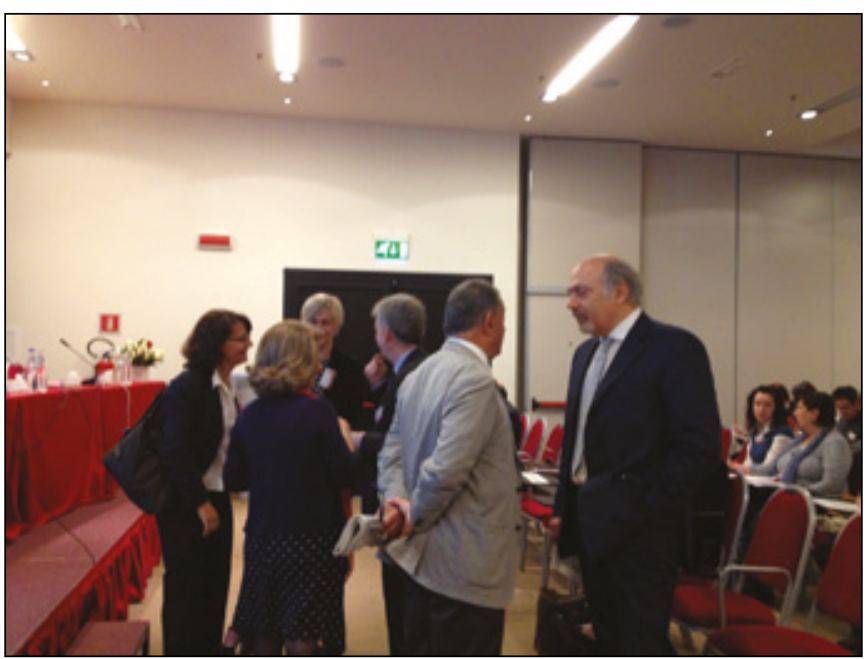

Fig. 3 - Dr.ssa A. Boletta, Dr.ssa A. Zatti, Prof. P. Messa, Prof. F. Scolari, Prof. A. Carrassi e, di spalle, L. Sternfeld.

non riuscendo a utilizzarlo quale fonte energetica, smettono di proliferare. Si suppone, inoltre, che il medesimo meccanismo antiproliferativo possa risultare efficace anche sulle cisti epatiche. I risultati di questo studio, anche se preliminari, risultano molto promettenti e fanno ben sperare in un utilizzo del 2-DG in un primo trial clinico (Fig. 3).

Successivamente, il Prof. Scolari (nefrologo), il Prof. Cenzato (neurochirurgo) e il Dr. Boccardi (neuroradiologo) hanno fatto il punto sull'incidenza di aneurismi intracranici nei pazienti con rene policistico. In una vivace discussione accademica, è emerso che gli aneurismi intracranici di meno di $7 \mathrm{~mm}$ sono molto frequenti anche nella popolazione generale, ma solo una bassissima percentuale di questi si rompe dando luogo a emorragie subaracnoidee. Tuttavia, i pazienti con rene policistico hanno un rischio aumentato di circa 5 volte di sviluppare aneurismi rispetto alla popolazione generale. Il trattamento degli aneurismi può essere chirurgico (tramite l'applicazione di clip) o endovascolare (tramite l'inserimento di piccole spirali di titanio che vanno a "tappare" l'aneurisma). È stato sottolineato, comunque, che, anche nei pazienti con rene policistico, la probabilità di rottura di aneurismi inferiori a $7 \mathrm{~mm}$ è la stessa della popolazione generale, molto bassa, e pertanto lo screening è necessario solo in presenza di familiarità.

Nella terza sessione, si è parlato del trapianto di fegato e di alcuni trattamenti per la riduzione del volume epatico in caso di grande ingombro sterico. È stato sottolineato il fatto che, contrariamente a quanto succede con il rene, anche un gran numero di cisti a carico del fegato non porta quasi mai a un'insufficienza epatica. Di conseguenza, la necessità di un trapianto è legata più alle conseguenze del grande accrescimento dell'organo che alla perdita di funzionalità. In alcuni casi, il fegato può crescere di molto (fino a $10 \mathrm{Kg}$, quando un fegato normale si aggira intorno al chilo e mezzo). Si è poi specificato che i trattamenti di resezione di parte del fegato (quando possibili e quando l'organo è molto ingrossato) non sono sempre consigliabili, prima di tutto perché non sono risolutivi (a causa delle recidive) e poi perché potrebbero portare

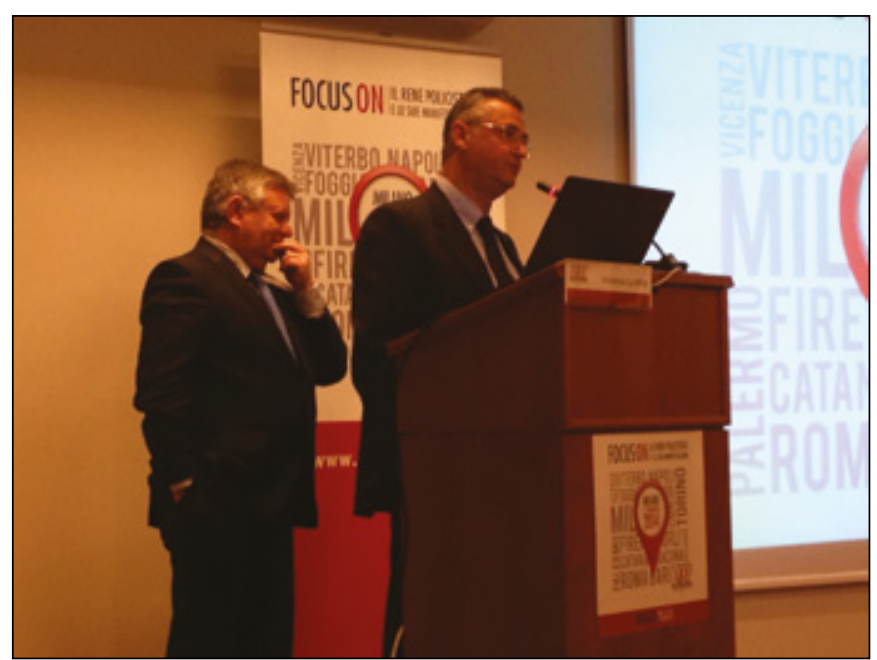

Fig. 4 - Prof. A. Santoro, Dr. V. La Milia.

a un aggravamento delle condizioni cliniche generali del paziente (infezioni, aderenze, ecc.), che potrebbe non sopportare un eventuale successivo trapianto.

Il Prof. Santoro e il Dr. La Milia hanno, quindi, trattato il tema delle terapie sostitutive: rispettivamente, emodialisi e dialisi peritoneale. Di queste sono stati spiegati i metodi, i rischi (ipotensione, aritmia e sclerotizzazione delle vene per l'emodialisi, infezioni e diminuzione della capacità filtrante della membrana peritoneale per la dialisi peritoneale), nonché le misure da adottare per la prevenzione di tali rischi (Fig. 4).

Nella sessione dedicata al trapianto di rene, i Professori Messa e Stratta hanno parlato delle varie problematiche inerenti al trapianto in pazienti con reni policistici. In particolare, hanno evidenziato la possibilità di effettuare la nefrectomia, sia mono che bilaterale, pre-durante o post-trapianto, a seconda delle diverse manifestazioni cliniche e delle esigenze chirurgiche. E stato interessante sapere che, nonostante il malato di rene policistico arrivi al trapianto a un'età mediamente più giovane (circa 10 anni prima) rispetto ai pazienti con altre patologie renali, l'aspettativa di vita post-trapianto e la sopravvivenza d'organo sono migliori.

La sessione forse più "pratica" per noi pazienti è stata quella tenuta dal Prof. Gesualdo su stili di vita e alimentazione. Gesualdo ha elencato le diverse problematiche legate alla patologia (tra cui: ipertensione, iperuricemia, calcolosi, proteinuria, ecc.) per poi indicare alcuni consigli di "buona alimentazione" (insieme alla convivialità!), per mantenere il più a lungo possibile la funzionalità renale e, quindi, per allontanare nel tempo l'insorgenza di insufficienza renale. Tra questi: limitare i cibi ricchi di conservanti (indicati nelle etichette degli alimenti come "E...") e gli insaccati, evitare bibite, alcol e fumo e consumare con moderazione carne e formaggi. Si possono, invece, mangiare con più libertà frutta e verdura e il pesce può essere consumato anche fino a tre volte a settimana. È stato, quindi, sottolineato come l'assunzione di proteine possa derivare anche dai legumi (che possono sostituire la carne, almeno qualche volta). Il Prof. ha poi indicato l'importanza di bere molta acqua (più di 2 litri al giorno), di limitare il condimento 


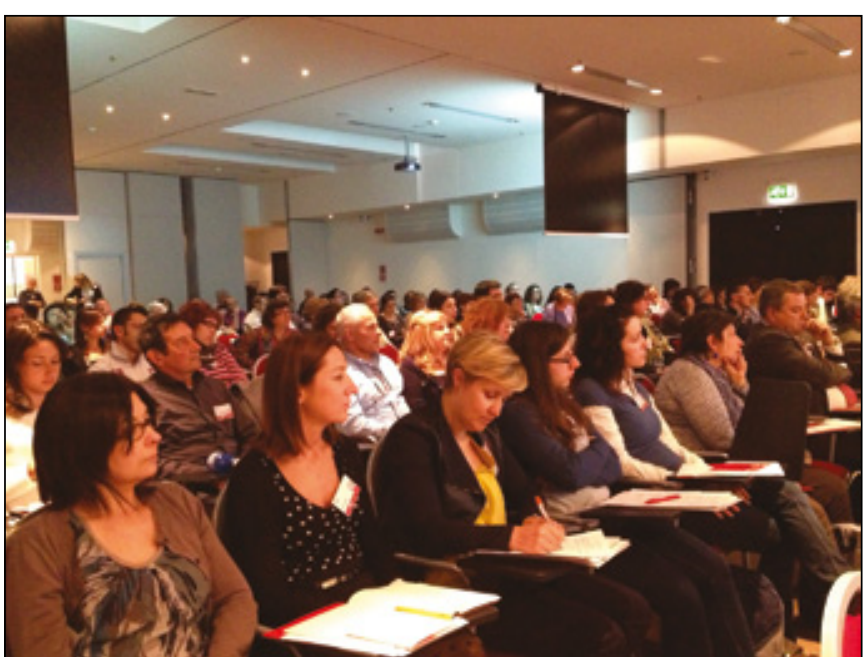

Fig. 5

degli alimenti (sale) e, soprattutto, di fare attività fisica, che va sempre abbinata a un'alimentazione sana. Ci ha dato sollievo sentir dire dal Prof. Gesualdo che non c'è nulla che non si possa mangiare: tutto dipende dalla quantità! Tutti questi preziosi consigli saranno prossimamente raccolti in un terzo libro AIRP, alla cui pubblicazione stiamo già lavorando. Infine, la Dr.ssa Massella ha parlato del rene policistico nel bambino sia autosomico dominante, ma anche nella più aggressiva forma autosomica recessiva, presentando diversi casi clinici e spiegando le problematiche di intervento nei pazienti pediatrici. In particolare, è stato interessante notare come la semplice, ma importante misurazione della pressione nei pazienti pediatrici non sia così scontata, bensì debba sempre essere rapportata al percentile di crescita del bambino.

In conclusione, durante la Tavola Rotonda (Fig. 5) è stata data la possibilità ai pazienti di fare domande ai Relatori e di esprimere dubbi o portare la propria testimonianza. In particolare, ci si è soffermati sul problema del dolore renale cronico e di come questo sia tanto disabilitante per il paziente quanto a volte poco considerato dal medico.

... Insomma, una giornata ricca di approfondimenti.

Desidero ora annunciarvi che, da quest'anno, AIRP si occuperà anche di $\mathrm{ARPKD}$.

Stiamo organizzando, quindi, un altro importantissimo appuntamento, fortemente voluto da AIRP per rispondere a un'esigenza molto sentita: il primo incontro nazionale con le famiglie di bambini affetti da rene policistico autosomico recessivo, Autosomal recessive polycystic kidney disease $(A R P K D)$, una malattia considerata "rara" e per la quale, perciò, è relativamente difficile reperire le informazioni che i genitori vorrebbero conoscere in modo approfondito.

L'incontro si terrà a Roma il 27 Settembre 2014 (Fig. 6), con il titolo "ARPKD chiama. AIRP risponde" e vedrà la presen-

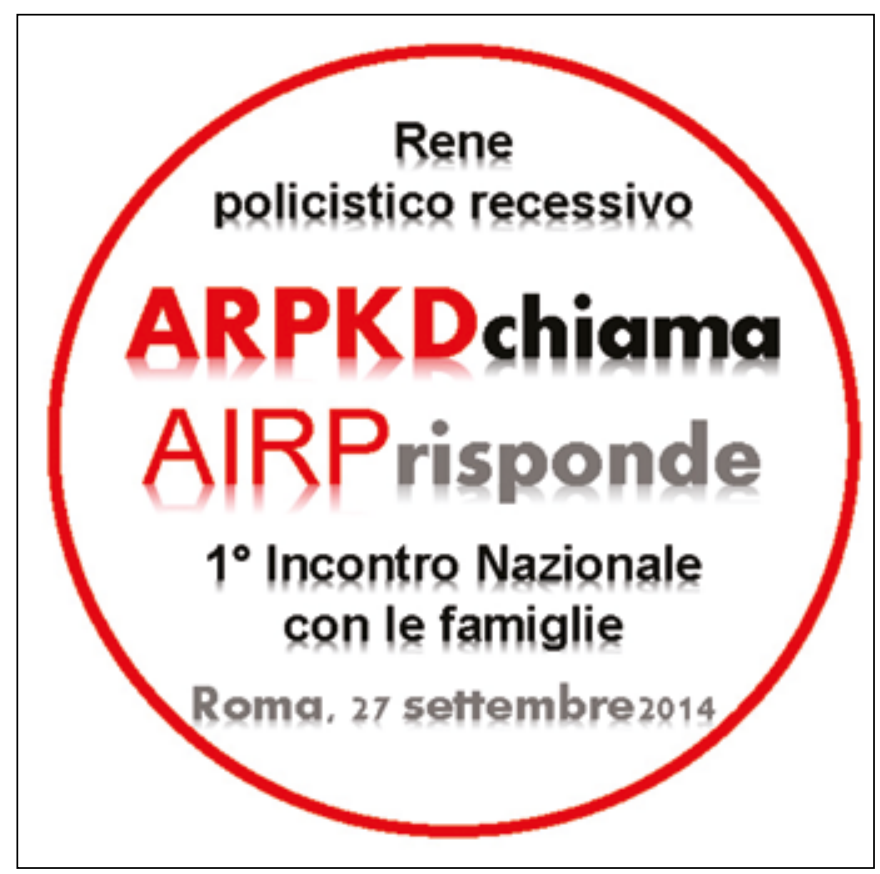

Fig. 6

za di nefrologi, genetisti e pediatri [saranno presenti Alessandra Boletta (ricercatrice), Laura Massella (pediatra nefrologa), Giovanni Montini (pediatra nefrologo), Salvatore Melchionda (biologo), Claudia Izzi (genetista), Gianfranco Savoldi (biologo) e Francesco Scolari (nefrologo)], che presenteranno contributi scientifici e clinici sulla patologia.

Ma non solo. Le famiglie dei piccoli pazienti potranno dialogare con questi medici, fare domande, chiarire dubbi e speriamo placare almeno in parte le loro comprensibili ansie. I dettagli dell'appuntamento saranno pubblicati sul nostro sito www.renepolicistico.it. Sono certa che molti risponderanno all'invito, ma, intanto, segnate sulla vostra agenda la data!

È in programma anche di aprire una pagina su EURORDIS, un portale internazionale dove vengono riportate tutte le malattie rare, ma, poiché manca l'ARPKD, sarà nostra cura attivarci per poter mettere on-line al più presto questa patologia anche in collaborazione con PKD International. Attiveremo un gruppo chiuso o una pagina su Facebook, così come lo abbiamo per ADPKD, e aggiorneremo il sito con una sezione dedicata.

Continuate a seguirci e a sostenerci!

Un caro saluto a tutti e ci vediamo a Settembre!

Luisa Sternfeld Pavia 